\title{
Methodology for Investigation of Risk Based Maintenance (MIRBA) for Mobile Mooring System
}

\author{
${ }^{1}$ Silvianita, ${ }^{2}$ Mohd. Faris Khamidi, ${ }^{1}$ Suntoyo and ${ }^{1}$ Dirta Marina Chamelia \\ ${ }^{1}$ Department of Ocean Engineering, Faculty of Marine Technology, \\ Institute Teknologi Sepuluh Nopember, 60111 Surabaya, Indonesia \\ ${ }^{2}$ Department of Built Environment, University of Reading Malaysia, Johor Bharu, Malaysia
}

\begin{abstract}
This study briefly presents MIRBA (Methodology for Investigation of Risk Based Maintenance) for mobile mooring system. MIRBA is developed based on bow tie analysis and AHP. Bow tie analysis consists of FTA (Fault Tree Analysis) on left part and ETA (Event Tree Analysis) on the right part. FTA is useful to determine the potential causes from critical top event until the undesired events are obtained. ETA (Event Tree Analysis) is helpful to define the possible consequence by relating an initiating event to various consequence models. MIRBA consists of risk management through bow tie analysis, risk mitigation by developing the mitigation plan and risk based maintenance using AHP. MURBA is aimed to provide a guidance on systematic methodology of risk based decision making which is useful to manage and reduce the risk for offshore platforms.
\end{abstract}

Key words: Decision, investigation, methodology, maintenance, risk, event tree analysis

\section{INTRODUCTION}

This study investigates the risk based decision making of mooring systems. Floating platform uses mooring systems for positioning and station keeping. Mooring system failure can cause disastrous damage to the platform. Mooring system failure can be caused by anchor failure (HSE, 2009). The anchor failure is associated with anchor/anchor line, mooring devices, winching equipment or fairleads (e.g., anchor dragging, breaking of mooring lines, loss of anchors and winch failures). Anchor failure is an important issue in mooring the floating units because of its function for station keeping. The platform may lose its position, adrift or even collapse. This knowledge has raised the need to perform risk based decision making which can be useful for oil and gas industry to examine the worst case scenarios.

The approaches of risk based decision making used in this study consist of FTA (Fault Tree Analysis), ETA (Event Tree Analysis) and AHP (Analytic Hierarchy Process). The objectives of this study are trying to combine those methods which are called MIRBA (Methodology for Investigation of Risk Based Maintenance). These methods are very useful in order to determine the risk analysis in offshore operation and maintenance (ABS, 2001; API, 1993). These methods have been applied in many areas, especially in offshore operation, risk assessment and maintenance (Table 1). In order to develop MIRBA, the following steps need to be taken:

- Developing the risk matrix based on frequency and consequence

- Determining the mitigation plan to reduce the risk

- Generating the best maintenance strategy selection on the basis of consequence using AHP

MIRBA application: The overviews of each steps in MIRBA are as follows.

MIRBA step 1; Building the complete bow tie: A bow tie is the combination method of fault tree analysis on the left and event tree analysis on the right. These bow ties are the basis for the application of the MIRBA methodology.

MIRBA step 2; Determining frequency of occurrence: The frequency of occurrence is considered for bow ties by using the same frequency for the critical event in FTA and ETA. First, it is needed to make an estimation of the frequency of occurrence in FTA on the basic event or undesired event based on the expert judgment. Then, each branch and gate combination events are solved and explored using Boolean algebra. Finally, all the parameters are passed on to the higher level to calculate the

Corresponding Author: Silvianita, Department of Ocean Engineering, Faculty of Marine Technology, Institute Teknologi Sepuluh Nopember, 60111 Surabaya, Indonesia 
Res. J. Applied Sci., 13 (1): 26-33, 2018

Table 1: Characteristics and application of bow tie and AHP

\begin{tabular}{lll} 
& MIRBA & \\
Critical views & Bow tie analy sis & Analytic Hierarchy Process (AHP) \\
Qualitative & $\checkmark$ & $\checkmark$ \\
Quantitative & $\checkmark$ & - \\
DNV & $\checkmark$ & - \\
ABS (2001) & - & - \\
API (1993) & Fowler (2003), Delvosalle et al. (2005), & Shafiq (2010) Bertolini and Bevilacqua (2006), \\
Applications in offshore & Cockshott (2005), Silvianita and Kurian (2013) & Dey (2001), Dawotola et al. (2011), \\
Risk assessment and reliability & Gowland (2006), Badreddine and Amor (2010) & Arunrai and Maiti (2010) \\
\hline
\end{tabular}

frequency of the top event. Once finished with the FTA, the frequency of top event is put in ETA to calculate the possible outcome. The outcome frequency based on expert judgments for each path progress to the right branching is calculated continually using the rules of Boolean algebra.

MIRBA step 3; Calculating the class of outcomes: Once the outcome frequency has been identified, then the class of outcome of hazardous phenomena is carried out. There are four points of view to determine the class of outcome that involves aspects of people, assets, environment and reputation. Each aspect has six levels to choose by the expert judgment in order to identify the outcome class.

MIRBA step 4; Developing the risk matrix: The risk matrix graph is constructed based on the frequency as $\mathrm{x}$-axis and outcome as the $\mathrm{y}$-axis. Four zones of risk level are classified in the risk matrix. Through the risk graph, the risk level can be seen and it will be easier to control and manage the risks.

MIRBA step 5; Determining the mitigation plan: Developing the mitigation plan is based on the highest risk leveland by doing so, the cost will be more efficient and effective. Mitigation plan is established for each of the undesired events on how to handle and manage the risk on daily basis.

MIRBA step 6; Determining the maintenance strategy: Maintenance strategy is developed in order to manage the risk failure. The method that is used to select the best maintenance is AHP as it is one of the most widely used of multi criteria decision making methods (Ling, 2001).

MIRBA step 6.1; Starting AHP by selecting the goal/objective: This step is the starting point of AHP procedure in order to prioritize the best maintenance plan for the mooring system. Construction of hierarchy in AHP is beginning with system identification by selecting the goal/objective.

MIRBA step 6.2; Developing the hierarchy tree: The hierarchical structure is developed based on the system identification that divided into several levels. Generally, AHP involves three levels:

- First level: description of the goal that need to be achieved

- Second level: the criteria of the factory to enable the goal to be achieved

- Third level: the alternative or the choices of the system in order to achieve the goal

MIRBA step 6.3; Calculating the matrix pair wise comparison: The hierarchy evaluation starts with the calculation of the matrix pair wise comparison. The judgment is made on a numerical scale ranging from 1-9.

MIRBA step 6.4; Calculating the priority vector: The priority vector describes the preference, importance or the likelihood of its elements with respect to a certain criteria. The priority vector is obtained from normalized eigenvector of the matrix.

MIRBA step 6.5; Selecting the alternative of choice: Once the calculation process through pair wise comparison of each element relative to the overall goal produces a goal priority vector, then, the consistency ratio throughout the matrix is checked. The consistency ratio must be $<0.1$

MIRBA step 7; Establish the maintenance strategy: The last step in MIRBA is to establish the maintenance strategy and action. This is important to control and manage the risk by understanding their individual risks and knowing the alternatives.

\section{RESULTS AND DISCUSSION}

Bow tie result: A bow tie is the combination method of fault tree analysis on the left and event tree analysis on the right. These bow ties are the basis for the application of the MIRBA methodology.

Bow tie analysis is developed for each critical event investigated in mooring system failure which are Mooring Line Breakage (MLB), Anchor Failure (AF), Appurtenances Connection Failure $(\mathrm{ACF})$ and Anchor Handling Failure (AHF), the detail process of mooring 
Res. J. Applied Sci., 13 (1): 26-33, 2018

\begin{tabular}{llll} 
Table 2: Frequency index & & F (per ship year) \\
\hline FI & Frequency & Definition & 10 \\
5 & Frequent & Likely to occur once per month on one ship & $10^{-1}$ \\
3 & $\begin{array}{l}\text { Reasonably probable } \\
\text { Remote }\end{array}$ & $\begin{array}{l}\text { Likely to occur once per y ear in a fleet of ships, i.e., likely to occur several times during a ships life } \\
\text { Likely to occur once per year in a fleet of } 1000 \text { of ships, i.e., } 10 \% \text { chance of occurring in the life } \\
\text { of } 4 \text { similar ships } \\
\text { Likely to occur once in } 100 \text { y ears in a fleet of } 1000 \text { ships, i.e., } 1 \% \text { chance of occurring in the life } \\
\text { of } 40 \text { similar ships }\end{array}$ & $10^{-3}$ \\
1 & Extremely remote & $10^{-5}$ & \\
\hline
\end{tabular}

Table 3: Class of consequences

Consequences

\begin{tabular}{lllll} 
Severity rating & People & Assets & Environment & Reputation \\
0 & Zero fnjury & Zero damage & Zero effect & Zero impact \\
1 & Slight fnjury & Slight damage & Slight effect & Slight impact \\
2 & Minor fnjury & Minor damage & Minor effect & Minor impact \\
3 & Critical fnjury & Critical damage & Critical effect & Critical impact \\
4 & Single fatality & Critical damage & Critical effect & Critical national impact \\
5 & Multiple fatalities & Extensive damage & Massive effect & Critical international impact \\
\hline
\end{tabular}

system failure were explained by Silvianita and Kurian (2013). The bow tie diagrams help to understand clearly through graphical visualization the relationship between the potential causes and their possible consequences. The bow tie diagram of AF summarizing the fault tree of $\mathrm{AF}$ in the left part and event tree of $\mathrm{AF}$ in the right part can be seen detail by Khamidi et al. (2016)

MIRBA step 2; Determining frequency of occurrence: The frequency of occurrence derives from the result of FTA which is then used as the frequency of initiating event in ETA. The frequency of initiating events multiplied by the pivotal events will result in the frequency of occurrence of the outcomes. Generally, there are several possible outcomes deriving from the initiating event followed by the pivotal events which include the success or failure possibility. The frequency of pivotal events is obtained from the expert's judgments based on their knowledge and experiences. Once the event tree diagram is constructed, the frequency of occurrence can be applied to the diagram for each path.

MIRBA step 3; Calculating the class of outcomes: Once the frequency of the outcome has been identified, the next step is to determine the class of the outcomes based on the DNV standards. There are four points of view to determine the class of outcome involving aspects of people, assets, environment and reputation. Each aspect has six levels to be chosen by the expert judgment in order to identify the outcome class.

MIRBA step 4; Developing the risk matrix: The risk matrix graph is constructed based on the frequency as $\mathrm{x}$-axis and outcome as the $\mathrm{y}$-axis. Four zones of risk level are classified in the risk matrix. Risk graphs describe the risk level for each event which shows the impact ratings.

Risk matrix is developed based on bow tie diagram and class of consequence categorization. The Risk Index
(RI) is developed according to the Frequency Index (FI) as shown in Table 2 developed by IMO (1997) and the Severity Index (SI) as seen in Table 3 developed by Dnv . Since, the frequency in ISO 17776 developed by DNV are descriptive in nature, therefore, this study adopts both standards, namely the frequency based on IMO and the consequence based on ISO. The risk matrix used in this study is a 7 by 6 risk matrix which useful to increase the visibility of risk level.

Class of consequences is divided into four criteria consisting of people, assets, environment and reputation of the company. All the possible outcomes of the bow tie analys is are estimated using this class of consequences and places in the risk matrix.

Based on both frequency index and class of consequence, the risk matrix mapping reveals the decision classes as being very high, high, medium and low. Table 4 explains the risk matrix classes that will be useful to generate the graphs of risk level.

The next step is to describe the risk level into risk graph. The risk graph characterizing both frequency and consequences of an event is helpful to serve as a communication tool to describe the risk level. The frequency of occurrence has been developed through the FTA and ETA resulting several potential outcomes of the mooring system. Furthermore, the class of consequences of the potential outcomes of mooring system needs to be found. Currently, there are insufficient data to determine the class of consequence, hence, it can be estimated through the Expert Opinion Survey (EOS). The expert estimates the class of consequence for each of the possible outcomes based on Table 3 .

Figure 1 shows the risk matrix of anchor failure with all the possible outcomes. The safe anchor is classified as medium level in the risk matrix graphs. Since, the anchor is located in the medium level, it is important to develop the mitigation plans. The other outcomes of $\mathrm{AF}$ are located in lower level which can be neglected. 
Res. J. Applied Sci., 13 (1): 26-33, 2018

\begin{tabular}{|c|c|c|c|c|c|c|}
\hline Severity/frequency & Negligible & Slight & Minor & Critical & Critical & Catastrophic \\
\hline Frequent & High & Very high & Very high & Very high & Very high & Very high \\
\hline Probable & Medium & High & High & Very high & Very high & Very high \\
\hline Reasonably probable & Medium & High & High & Very high & Very high & Very high \\
\hline Occasional & Medium & Medium & Medium & High & High & Very high \\
\hline Remote & Low & Medium & Medium & Medium & High & High \\
\hline Improbable & Low & Low & Low & Medium & Medium & High \\
\hline Extremely remote & Low & Low & Low & Low & Medium & Medium \\
\hline
\end{tabular}

Table 5: The EOS for mitigation plans

\begin{tabular}{|c|c|c|}
\hline Basic events & Codes/Mitigation codes & Mitigation plans \\
\hline \multirow[t]{5}{*}{ Design error } & $\mathrm{DE}$ & \\
\hline & M1 & Carry out pre project arrangement to reduce design errors \\
\hline & M2 & $\begin{array}{l}\text { Design to be reviewed and validated by } 3 \text { rd party certification (ABS or } \\
\text { DNV or lloy ds, etc.) }\end{array}$ \\
\hline & M3 & Review the design plan with independent engineering/the consultant \\
\hline & M4 & Obtain design liability insurance \\
\hline \multirow{4}{*}{ Human error } & $\mathrm{HE}$ & \\
\hline & M1 & Trained the crew regularly to maintain the skills \\
\hline & M2 & Hired the certified and credible crew \\
\hline & M3 & Employ competent crew with the certification \\
\hline \multirow[t]{4}{*}{ Improper quality control } & IQC & \\
\hline & M1 & Implement proper quality control procedures and supervision \\
\hline & M2 & $\begin{array}{l}\text { Employ all regulatory documentation including vessel classification, } \\
\text { equipment and personnel certification }\end{array}$ \\
\hline & M3 & Engage 3rd party inspection \\
\hline \multirow[t]{4}{*}{ Incompetence crews } & $\mathrm{IC}$ & \\
\hline & M1 & The crew regularly trained to maintain the skills \\
\hline & M2 & Hired the certified and credible crew \\
\hline & M3 & Employ competent crew with the certification \\
\hline \multirow[t]{3}{*}{ Mechanical failure } & MF & \\
\hline & M1 & $\begin{array}{l}\text { Conduct the verification test to examine the performance of mechanical } \\
\text { mooring hardware }\end{array}$ \\
\hline & M2 & Ensure mechanical condition of the winches \\
\hline \multirow[t]{3}{*}{ Rocky seabed } & RS & \\
\hline & M1 & Conduct comprehensive survey on sea bed location \\
\hline & M2 & Review and verify adequacy/suitability of anchoring design \\
\hline \multirow[t]{6}{*}{ Wrong material } & WM & \\
\hline & M1 & Revisit/verify correctness of design and construction specifications \\
\hline & M2 & Engage 3rd party verification during manufacturing and construction \\
\hline & M3 & Verify all mill certificates \\
\hline & M4 & Ensure proper NDT program and adequate record procedure for traceability \\
\hline & M5 & Improve the quality control in manufacturing process \\
\hline
\end{tabular}

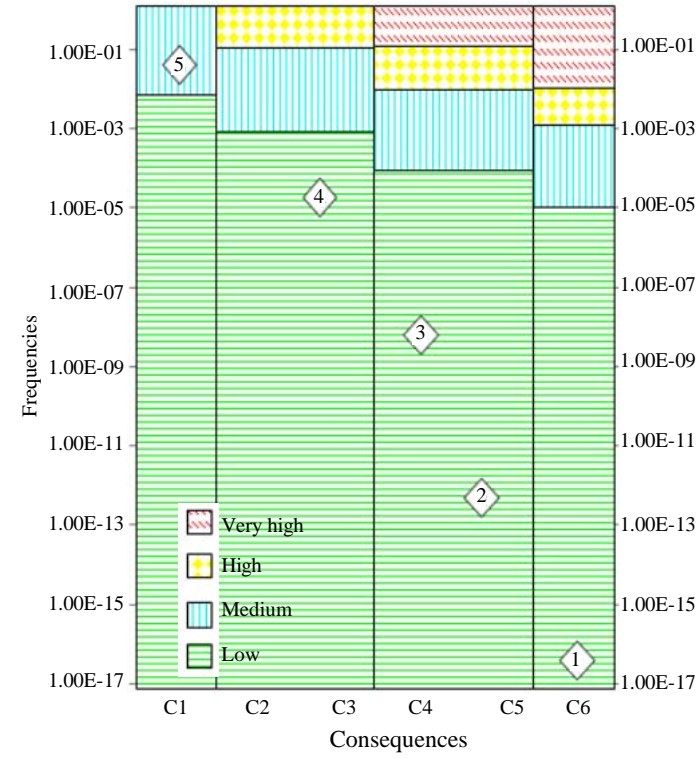

Fig. 1: Risk matrix of $\mathrm{AF}$, outcomes $(1,2,3,4,5)$
MIRBA step 5; Determining the mitigation plan: Developing the mitigation plan is based on the risk level. It is useful to minimize the failure. Mitigation plan is established for each of the undesired events on how to handle and manage the risk. The bow tie framework helps to identify all the related causes of an event and their consequences. By identifying all the causes of possible failure, the list of mitigation plans can be established. Mitigation plans are developed based on the result of the risk level of the bow tie analysis. With regard to the risk level classification the four critical events of mooring system failure, $\mathrm{MLB}, \mathrm{AF}, \mathrm{ACF}$ and $\mathrm{AHF}$ are in the medium level. Therefore, it is important to establish mitigation plans for each of undesired events. The mitigation plans are obtained based on all the critical basic events in mooring system failure. Table 5 shows the mitigation plans for some of basic events.

AHP results: AHP starts by selecting the goal/objective through the hierarchical structure. Hierarchical structure is used to model the problem which contains the decision 


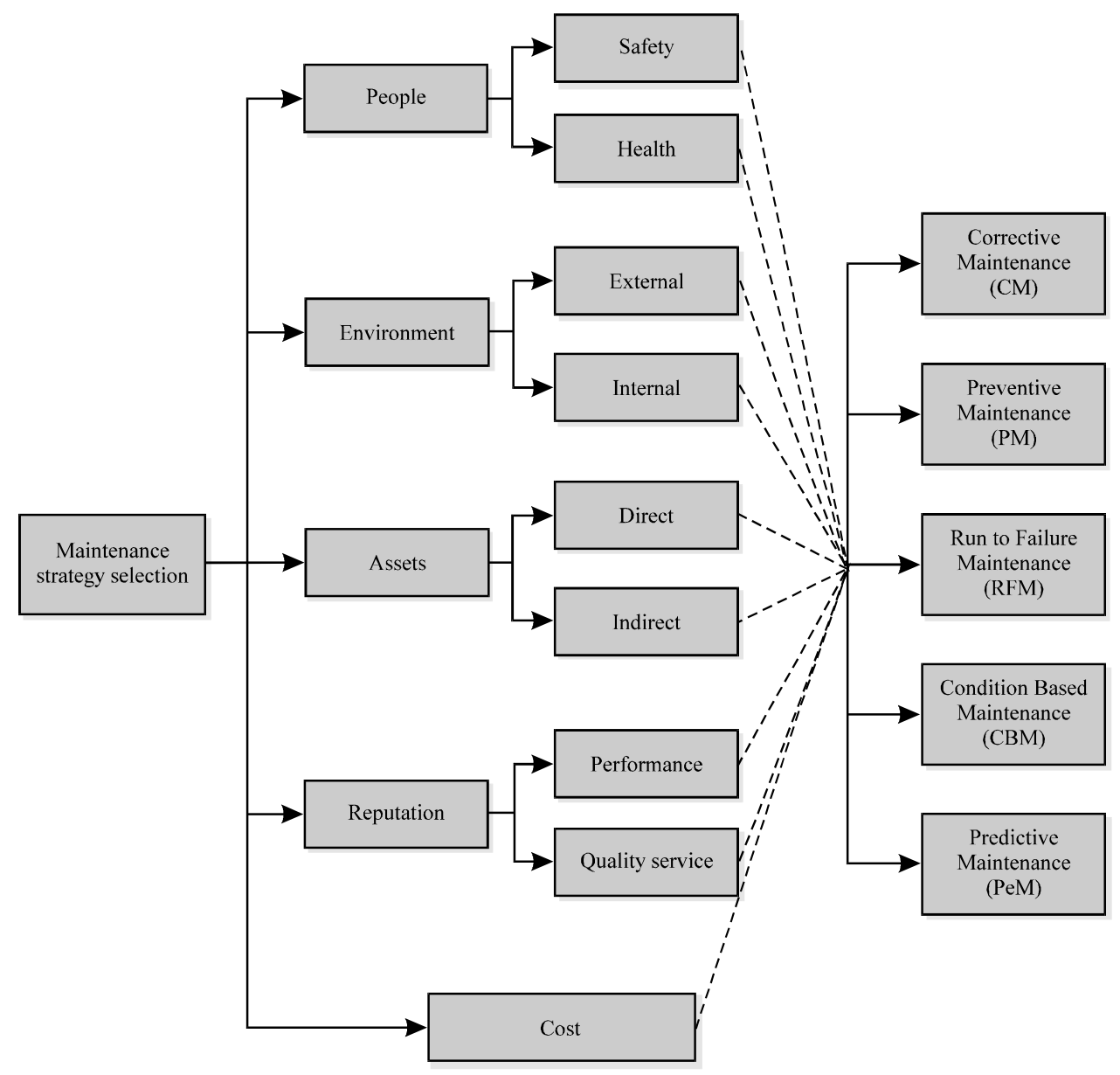

Fig. 2: Maintainance strategy for mooring system on the basis of consequence of failure

goal, the alternative to achieve it and the criteria to evaluate the alternatives. The goal of this study is to select the best maintenance strategy for the mobile mooring system.

MIRBA step 6; Determining the maintenance strategy: Maintenance strategy is developed in order to maintain the risk failure. Selecting the best maintenance strategy is cost saving because it will be more efficient and effective. The method used to select the best maintenance strategy is AHP. It is one of the most widely used of multi criteria decision making methods (Ling, 2001). In order to apply the analytic hierarchy process, few steps need to be taken which include construction of hierarchical tree, evaluation of hierarchy and sensitivity analysis. This study uses AHP to select the best maintenance strategy for mooring system on the basis of the consequences of failure as shown in Fig. 2.

MIRBA step 6.1; Starting AHP by selecting the goal/objective: AHP starts by selecting the goal/objective through the hierarchical structure. Hierarchical structure is used to model the problem which contains the decision goal, the alternative to achieve it and the criteria to evaluate the alternatives. The goal of this study is to select the best maintenance strategy for the mobile mooring system.

MIRBA step 6.2; Developing the hierarchy tree: The hierarchical structure is used to model the decision in a systematic way. The design of the hierarchical structure is based on knowledge, judgments and opinion of the experts involved in decision making process. Hierarchical structure is visualized through a diagram tree showing the goal at the first level, criteria at the second level, sub criteria at the third level and the alternative at last level.

Construction of the hierarchy tree: Construction of hierarchy in AHP is started from system identification and hierarchical structure. System identification in AHP helps the decision maker understand their problem and find the 
Res. J. Applied Sci., 13 (1): 26-33, 2018

Table 6: The fundamental scale of absolute numbers (Thomas, 1988)

\begin{tabular}{|c|c|c|}
\hline Scales & Definitions & Description \\
\hline 1 & Equal importance & Two activities contribute equally to the objective \\
\hline 2 & Weak or slight & \\
\hline 3 & Moderate importance & Experience and judgment slightly favor one activity over another \\
\hline 4 & Moderate plus & \\
\hline 5 & Strong importance & Experience and judgment strongly favor one activity over another \\
\hline 6 & Strong plus & \\
\hline 7 & Very strong or demonstrated importance & $\begin{array}{l}\text { An activity is favor very strongly over another, its dominance demonstrated } \\
\text { in practice }\end{array}$ \\
\hline 8 & Very, very strong & \\
\hline 9 & Extreme importance & $\begin{array}{l}\text { The evidence favoring one activity over another is of the highest possible order } \\
\text { of affirmation }\end{array}$ \\
\hline Reciprocals of above & $\begin{array}{l}\text { If activity } i \text { has one of the above non zero numbers } \\
\text { assigned to it when compared with activity } j \text {, then } \\
j \text { has the reciprocal value when compared with } i\end{array}$ & A reasonable assumption \\
\hline $1.1-1.9$ & If the activities are very close & $\begin{array}{l}\text { May be difficult to assign the best value but when compared with other } \\
\text { contrasting activities the size of the small numbers would not be too noticeable, } \\
\text { yet, they can still indicate the relative importance of the activities }\end{array}$ \\
\hline
\end{tabular}

best solutions suitable for their goal. Hierarchical structure composes the decision problem into a hierarchy in order to comprehend sub problems easily and to evaluate its various elements by comparing them using AHP scale.

System identification: The first step in executing the AHP is to identify the systems to be employed in the maintenance strategy selection. This step includes the brainstorming with the experts aimed to acquire knowledge with the help of expert opinion survey and interviews.

Hierarchical structure: Based on the system identification, the information can be constructed to a hierarchy as shown in Fig. 2. The hierarchy of AHP generally consists of four levels as follows:

- First level is the goal that needs to be achieved

- Second level is criteria of the factor to enable the goal to be achieved

- The third level is sub factor of the factor in the previous level

- The fourth level is alternatives of the maintenance strategies

Figure 2 describes the hierarchical structure for maintenance strategy for mooring system on the basis of consequence of failure. The first level is the goal which is maintenance strategy selection on the basis of consequence. The second level is the factors that need to be considered in the consequence of mooring failure consisting of people, environment, assets, reputation and cost. The third level is the sub factor that can contribute to each factor of the consequence of mooring failures. The four factors and their sub factor on the basis of consequence are obtained based on the interview with the experts consisting of:

- People considering the safety and health of the personnel

- Environment by taking into account the external damage and internal damage of the platform

- Assets by considering the direct damage having tangible effects of the failure and indirect damage into account the possible reduction of the failure on the working life of the platform

- Reputation by considering the performance and quality service

- Costs, that can include the crew cost and spare past cost

MIRBA step 6.3; Calculating the matrix pair wise comparison: The hierarchy evaluation starts with the calculation of the matrix pairwise comparison. The judgment is made on a numerical scale ranging from 1-9 as shown in Table 6. The judgments of the relative importance of the elements with respect to the overall goal of the hierarchy tree are made. Elements at each level of the hierarchy are compared to each other in pairs with their respective parents in the next higher level.

AHP output for maintenance strategy on the basis of consequence: Table 7 shows AHP output for maintenance strategy on the basis of consequences. The hierarchical structure for maintenance strategy on the basis of consequence as shown in Fig. 2 is used to develop 
Res. J. Applied Sci., 13 (1): 26-33, 2018

Table 7: AHP output on maintenance strategy on the basis of consequences

\begin{tabular}{|c|c|c|c|c|c|c|c|c|}
\hline \multirow[b]{2}{*}{ Critical factors } & \multicolumn{3}{|c|}{ Maintenance factors } & \multicolumn{5}{|c|}{ AHP output on maintenance strategy } \\
\hline & Priority & Sub factor & Priority & $\mathrm{CM}$ & PM & RTF & CBM & PeM \\
\hline \multirow[t]{2}{*}{ People } & 0.460 & Safety & 0.269 & 0.031 & 0.070 & 0.021 & 0.050 & 0.098 \\
\hline & & Health & 0.192 & 0.038 & 0.035 & 0.016 & 0.037 & 0.067 \\
\hline \multirow[t]{2}{*}{ Environment } & 0.139 & External & 0.051 & 0.007 & 0.016 & 0.005 & 0.008 & 0.015 \\
\hline & & Internal & 0.087 & 0.011 & 0.028 & 0.011 & 0.015 & 0.023 \\
\hline \multirow[t]{2}{*}{ Assets } & 0.163 & Direct & 0.126 & 0.015 & 0.032 & 0.010 & 0.023 & 0.046 \\
\hline & & Indirect & 0.037 & 0.006 & 0.009 & 0.004 & 0.007 & 0.012 \\
\hline \multirow[t]{2}{*}{ Reputation } & 0.100 & Performance & 0.055 & 0.006 & 0.014 & 0.004 & 0.010 & 0.021 \\
\hline & & Quality S. & 0.046 & 0.005 & 0.012 & 0.004 & 0.008 & 0.017 \\
\hline Cost & 0.137 & & & 0.030 & 0.014 & 0.060 & 0.021 & 0.012 \\
\hline \multicolumn{4}{|c|}{ Priority of maintenance on the basis of consequences } & 0.147 & 0.235 & 0.126 & 0.179 & 0.313 \\
\hline \multicolumn{4}{|c|}{ Ranking } & 4 & 2 & 5 & 3 & 1 \\
\hline
\end{tabular}

eighth EOS. The results of the pair wise comparison of the first level of the hierarchy indicate that the highest consequences of mooring system will give impact to people namely $46 \%$. The second highest consequence of mooring system will impact to assets of $14.3 \%$. The third highest consequence will impact to environment of $13.9 \%$, to the maintenance cost $13.7 \%$ and to the reputation, image of the company of $10 \%$.

\section{CONCLUSION}

The study's objective is to develop Methodology for Investigation of Risk Based Maintenance (MIRBA). The following are the main findings related to the objectives: the risk matrix graphs represent the value of frequency which derive from FTA and consequence obtained from ETA. The combination of the FTA and ETA is called bow tie analysis. The risk matrix graphs show five possible consequences of the critical hazards of mooring system, namely mooring line, anchor, appurtenances connections and anchor handling. The mitigation plans are obtained based on all the critical basic events in mooring system failure as can be seen in Table 5 .

The best maintenance strategy selection is obtained based on Analytic Hierarchy Process (AHP). The highest priority maintenance strategy for mooring system on the basis of the consequence of failure is PeM with $31.3 \%$.

\section{ACKNOWLEDGEMENT}

We are very grateful to all respondents for the useful and helpful information. This research was supported by Dana Lokal ITS Tahun 2017 for Laboratory Research.

\section{REFERENCES}

ABS., 2001. Principles of risk based decision making. Canberra, Australia.
API., 1993. Recommended practice for design and hazards analysis for offshore production facilities. Application Programming Interface, Washington.

Arunraj, N.S. and J. Maiti, 2010. Risk-based maintenance policy selection using AHP and goal programming. Safety Sci., 48: 238-247.

Badreddine, A. and N.B. Amor, 2010. A dynamic barriers implementation in Bayesian-based bow tie diagrams for risk analysis. Proceedings of the 2010 IEEE/ACS International Conference on Computer Systems and Applications (AICCSA), May 16-19, 2010, IEEE, Hammamet, Tunisia, ISBN:978-1-4244-7717-3, pp: 1-8.

Bertolini, M. and M. Bevilacqua, 2006. A combined goal programming-AHP approach to maintenance selection problem. Reliab. Eng. Syst. Saf., 91: 839-848.

Cockshott, J.E., 2005. Probability bow-ties: A transparent risk management tool. Process Saf. Environ. Prot., 83: 307-316.

Dawotola, A.W., P.H.A.J.M.V. Gelder and J.K. Vrijling, 2011. Decision analysis framework for risk management of crude oil pipeline system. Adv. Decis. Sci., 2017: 1-17.

Delvosalle, C., C. Fievez, A. Pipart, J.C. Fabrega and E. Planas et al., 2005. Identification of reference accident scenarios in SEVESO establishments. Reliab. Eng. Syst. Saf., 90: 238-246.

Dey, P.K., 2001. A risk-based model for inspection and maintenance of cross-country petroleum pipeline. J. Qual. Maintenance Eng., 7: $25-43$.

Fowler, D., 2003. EUR RVSM POSC development FHA review report. Farrer Huxley Associates, London, England.

Gowland, R., 2006. The accidental risk assessment methodology for industries (ARAMIS)/layer of protection analysis (LOPA) methodology: A step forward towards convergent practices in risk assessment?. J. Hazard. Mater., 130: 307-310. 
HSE., 2009. Accident statistics for offshore units on the UKCS 1990-2007. UK Oil and Gas Industry Association Limited, London, England, UK.

IMO., 1997. Interim guidelines for the Application of Formal Safety Assessment (FSA) to the IMO rule making process. International Maritime Organization, London, England.

Khamidi, M., K. Sambodho, N. Syahroni, Y. Mulyadi and M. Zikra, 2016. Investigation of Risk Based Decision Making for Mobile Mooring System. In: Applied Mechanics and Materials, Sutardi, B.P., R.D.M. Abu-Ayyad and L. Jiing-Yih (Eds.). Trans Tech Publications, Switzerland, pp: 233-238.
Ling, X., 2001. Introduction to Multi Criteria Decision Making and the Evidential Reasoning Approach. University of Manchester, Manchester, UK., ISBN: 9781861151117 ,

Shafiq, N., 2010. Prioritizing the pipeline maintenance approach using analytical hierarchical process. Intl. Rev. Mech. Eng., 4: 346-352.

Silvianita, M.F.K. and V.J. Kurian, 2013. Decision making for safety assessment of mobile mooring system. Jurnal Teknologi Sciences Engineering, 61: 41-52.

Thomas, L.S., 1988. The Analytic Hierarchy Process. University of Pittsburgh Press, Pittsburgh, Pennsylvania, USA., ISBN:9780070543713, Pages: 440. 